\title{
Size Distributions of Cadmium Sulfide Nanoparticles Obtained from Templating Methods
}

\author{
Paulo J. G. Goutinho, Gandido A. G. Mendes, and Teresa S. V. Reis \\ Departamento de Física, Universidade do Minho, Campus de Gualtar, Braga, Portugal
}

\begin{abstract}
Cadmium sulfide (GdS) nanoparticles were obtained by soft templating methods using either an already established revered micelle route or a new procedure based on gel electrophoresis. The UVVis absorption or the photoluminescence excitation spectra were fitted using the GdS electronic structure available in the literature together with a size distribution. The obtained results indicate that the amount of sodium dodecyl sulphate as a component of the agarose gel formulation has a profound effect on the resulting nanoparticle population.
\end{abstract}

Key words: GdS nanoparticles; templating; size distribution; photoluminescence

\section{Introduction}

Nanotechnology is a field of very active investigation. There is a wide scope of technological applications ranging from photocatalysis and sensors to fluorescence imaging.

In this work, cadmium sulfide (CdS) nanoparticles have been prepared by templating methods using surfactants and gel electrophoresis. The surfactant templating protocol was based on that of Pileni, ${ }^{1}$ using AOT [sodium bis(2-ethylhexyl) sulfosuccinate] waterin-oil microemulsions. In the gel electrophoresis procedure, cadmium and sulfide ions migrate under an electric field in opposite directions until they meet and react within the gel pores. This reaction is thus controlled by diffusion through the gel pores. The application of reaction-diffusion processes in nanofabrication involving gels has recently been reviewed. ${ }^{2}$ The size dependence of CdS electronic states was obtained by a tight binding approximation. ${ }^{3}$ Using these theoretical results, in conjunction with a size distribution and a Mie formalism for the scatter/absorption of nanoparticles of a given size, we were able to fit the experimental absorption and excitation spectra of CdS nanoparticles, either in AOT reversed micelles or in dried gels. In the case of AOT templating, the resulting particles should be spherical and the calculated average sizes can be compared to those obtained using empirical relations between first absorption peak and nanoparticle

Address for correspondence: Paulo J.G. Coutinho, Departamento de Física, Universidade do Minho, Campus de Gualtar, 4710-057 Braga, Portugal. Voice: +351253 604321; fax: +351 253604061

pcoutinho@fisica.uminho.pt size proposed by $\mathrm{Yu}$ and colleagues. ${ }^{4}$ In the gel electrophoresis templating experiments, excess concentration of one of the ions and the presence of sodium dodecyl sulphate (SDS) surfactant was found to influence the size distribution of the nanoparticles and the corresponding photoluminescence spectra.

\section{Experimental}

Nanoparticles have been prepared by soft routes with mild chemical conditions using the water cavities in microheterogeneous media as templates. ${ }^{1,5}$ In this work we prepared CdS nanoparticles by two different methods.

In method 1, the water pools of reversed micellar systems ${ }^{1}$ were used as templates. In brief, solutions of reversed micelles containing either cadmium nitrate or sodium sulphide are added together. After $\sim 1$ min a yellow/orange color develops which is indicative of CdS formation.

In method 2 the pores of an agarose gel under an electric field are used as a templating medium. To our knowledge, it is the first time that the latter procedure has been used. In brief, using an electrophoresis apparatus, a sodium sulphide solution is inserted in a well near the negative pole and, in a corresponding well near the positive pole, a cadmium nitrate solution is inserted. The electric fieldinduced migration of the ions will eventually lead to their encounter and the formation of CdS particles within the gel pore. SDS was also included as its surface activity could influence the particle growth within the gel. 


\section{Modelization}

The absorption spectra of CdS quantum dots (spherical particles) of various sizes have been empirically described by Yu et al. ${ }^{4}$ with the following equation

$$
\begin{aligned}
d(\mathrm{~nm})= & -6.6521 \times 10^{-8} \lambda_{\max }^{3}+1.9557 \\
& \times 10^{-4} \lambda_{\max }^{2}-0.092352 \lambda_{\max }+13.29
\end{aligned}
$$

where $d$ is mean particle diameter and $\lambda_{\max }$ is the maximum wavelength of the first excitonic band. This equation will allow an estimation of the mean particle diameter, which will validate the modelization of the CidS nanoparticle absorption to be used.

Mie scattering theory applied to spherical partially absorbing particles gives ${ }^{6,7}$ :

$$
\sigma=12 \pi \frac{\omega}{c} \varepsilon_{m}^{3 / 2} R^{3} \frac{\varepsilon_{2}}{\left(\varepsilon_{1}+2 \varepsilon_{m}\right)^{2}+\varepsilon_{2}^{2}}
$$

where $\varepsilon_{m}$ is the static dielectric constant of the surrounding medium, $\varepsilon_{1}$ and $\varepsilon_{2}$ are the real and imaginary parts of the dynamic dielectric constant of $\mathrm{CdS}$ particles, $\mathrm{R}$ is the particle radius, $c$ is the velocity of light in a vacuum, and $\omega$ is the light frequency.

$\varepsilon_{1}$ and $\varepsilon_{2}$ depend on light frequency and on the light absorption characteristics of the particles through:

$$
\begin{gathered}
\varepsilon_{1}(\omega)=\varepsilon_{S}+\sum_{i} \frac{f_{i}\left(\omega^{2}-\omega_{i}^{2}\right)}{\left(\omega^{2}-\omega_{i}^{2}\right)^{2}+\omega^{2} \Gamma_{i}^{2}} \\
\varepsilon_{2}(\omega)=\sum_{i} \frac{f_{i} \omega \Gamma_{i}}{\left(\omega^{2}-\omega_{i}^{2}\right)^{2}+\omega^{2} \Gamma_{i}^{2}}
\end{gathered}
$$

where $\varepsilon_{\mathrm{S}}$ is the static dielectric constant of $\mathrm{CdS}$ and $\omega_{i}, \Gamma_{i}$, and $f_{i}$ represent, respectively, each transition's energy, broadening, and oscillator strength.

The CdS quantum dot transition energies are obtained using theoretical calculations by Fonoberov and colleagues. ${ }^{3}$ The exciton energy level $1 \mathrm{~S}_{3 / 2}$ is given by

$$
\mathrm{E}_{\mathrm{exc}}\left(1 \mathrm{~S}_{3 / 2}\right)=\mathrm{E}_{\mathrm{g}}+\mathrm{E}_{\mathrm{e} 0}(\mathrm{R})+\frac{706.0}{\mathrm{R}^{1.987}}-\frac{431.9}{\mathrm{R}}(\mathrm{meV})
$$

other levels are graphically reported ${ }^{3}$ as a function of particle radius, $R$, between $1 \mathrm{~nm}$ and $5 \mathrm{~nm}, \mathrm{E}_{\mathrm{g}}$ is the bulk CdS band gap, and $\mathrm{E}_{\mathrm{e} 0}$ is the quantum confinement electron ground state energy that is given by

$$
E_{e 0}(\mathrm{meV})=\frac{4886.16}{V^{2 / 3}}
$$

where $\mathrm{V}$ is the particle volume in $\mathrm{nm}^{3}$

Oscillator strengths were also graphically reported for four optically active exciton states $\left(1 \mathrm{~S}_{3 / 2}, 1 \mathrm{~S}_{1 / 2}\right.$, $2 \mathrm{~S}_{3 / 2}$, and $3 \mathrm{~S}_{3 / 2}$ ).

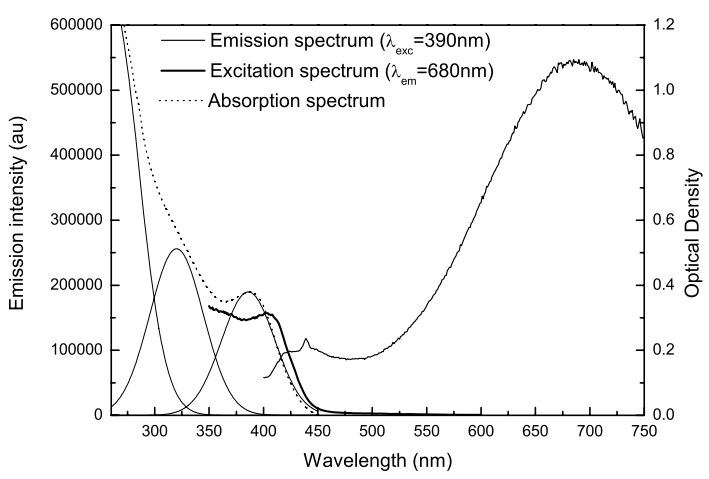

FIGURE 1. Emission, excitation, and absorption spectra of CdS nanoparticles obtained by reversed micelle templating $(\operatorname{method} 1)$ with $\omega_{\circ}=5.0$.

Other exciton levels can be constructed using higher quantum confinement electron energies. Fonoberov and co-workers, ${ }^{3}$ reported the first 20 electron energy levels. We assume that the form of EQUATION (5) is mantained substituting $\mathrm{E}_{\mathrm{e} 0}$ by the higher energy electron states. Also the same dependence of oscillator strengths on particle radius is assumed but multiplied by a constant factor $\mathrm{f}_{\mathrm{k}}$, where $\mathrm{k}$ runs from 1 to 6 corresponding to the six different quantum confinement electron energies reported by Fonoberov et al. ${ }^{3}$

\section{Results and Discussion}

In method 1 we expect that the resulting nanoparticles are of spherical shape. Through a Gaussian decomposition of the absorption spectra, the maximum absorption wavelength of the first excitonic band can be obtained. In turn, this value allows an estimation of the mean particle size using EQUATION(1).

In FIgURE 1 the emission, excitation, and absorption spectra of CdS nanoparticles obtained by method 1 with $\omega_{0}=5.0$ are plotted together with the Gaussian decomposition of the absorption spectra. The parameter $\omega_{0}$ is determined by the amount of water in the w/o AOT microemulsion:

$$
\omega_{\mathrm{o}}=\frac{\left[\mathrm{H}_{2} \mathrm{O}\right]}{[\mathrm{AOT}]}
$$

and is related to the size of the reversed micelles through $^{8}$ :

$$
R_{\mathrm{w}}(\AA)=1.5 \omega_{\mathrm{o}}
$$

Normalized absorption spectra of CdS nanoparticles obtained by reversed micelle templating 
TABLE 1. Fitting of CdS absorption spectra obtained by reversed micelle templating (method 1 )

\begin{tabular}{lcccccccccc}
\hline$\omega_{\mathrm{o}}$ & $\mathrm{R}_{\mathrm{W}}(\mathrm{nm})$ & $\mathrm{d} / 2^{a}(\mathrm{~nm})$ & $\mathrm{R}_{\mathrm{m}}{ }^{b}(\mathrm{~nm})$ & $\mathrm{R}_{\mathrm{av}}(\mathrm{nm})$ & $\sigma(\mathrm{nm})$ & $\mathrm{f}_{2} / \mathrm{f}_{1}$ & $\mathrm{f}_{3} / \mathrm{f}_{1}$ & $\mathrm{f}_{4} / \mathrm{f}_{1}$ & $\mathrm{f}_{5} / \mathrm{f}_{1}$ & $\mathrm{f}_{6} / \mathrm{f}_{1}$ \\
\hline 2.5 & 0.38 & 1.31 & 1.47 & 1.47 & 0.229 & 2.21 & 9.10 & 6.15 & 0.155 & 0.003 \\
5.0 & 0.75 & 1.48 & 1.60 & 1.60 & 0.255 & 2.01 & 5.53 & 2.46 & 22.9 & 0.00053 \\
7.5 & 1.1 & 1.50 & 1.53 & 1.52 & 0.356 & 1.63 & 3.65 & 2.29 & 20.3 & 0.94 \\
15 & 2.2 & 1.42 & 1.52 & 1.52 & 0,245 & 1.5 & 10.5 & 22.4 & 0.0039 & 0.0042 \\
\hline
\end{tabular}

${ }^{a}$ Obtained from EQUATION $(1)$.

${ }^{b}$ Mean particle size in the $1-5 \mathrm{~nm}$ range, calculated from a Gaussian distribution with $\mathrm{R}_{\mathrm{av}}$ average size and $\sigma^{2}$ standard deviation.

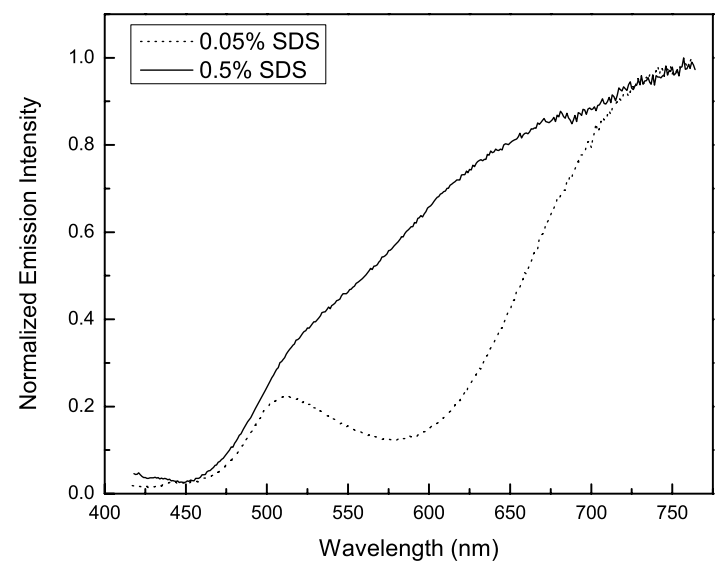

FIGURE 2. Emission spectra of $\mathrm{CdS}$ in dried agarose gels with $\lambda_{\text {exc }}=400 \mathrm{~nm}$.

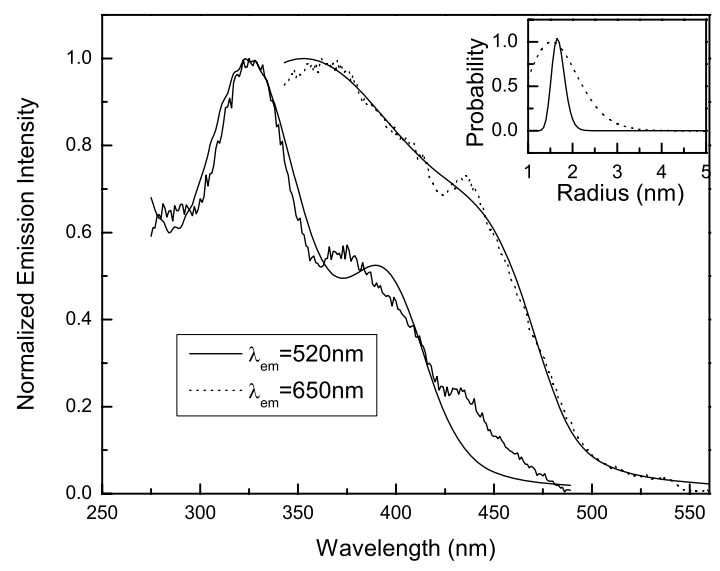

FIGURE 3. Excitation spectra of $\mathrm{CdS}$ in dried agarose gels with $[S D S]=0.005 \mathrm{M}$.

(method 1) were also fitted using Mie scattering/absorption theory (see MoDELIZATION section) together with a Gaussian size distribution function. In TABLE 1 the various fitted parameters together with the mean particle size, obtained by both models, are shown.

The average nanoparticle sizes obtained by the presented models are compatible. This serves as an initial

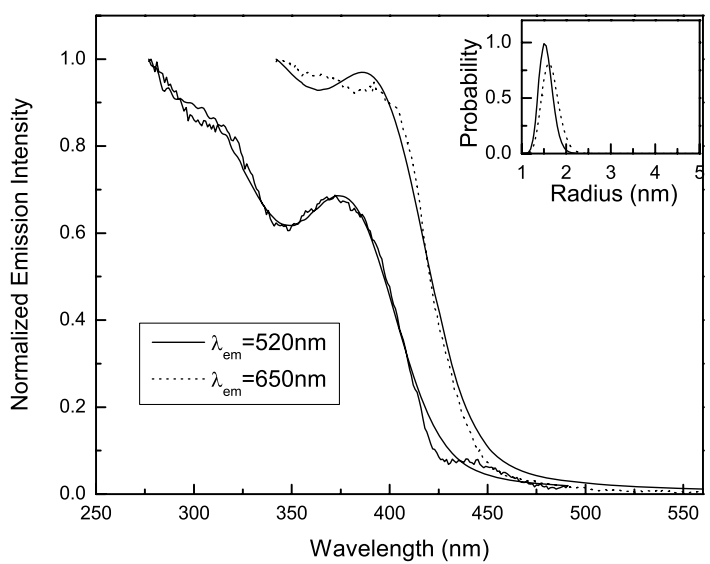

FIGURE 4. Excitation spectra of CdS in dried agarose gels with $[S D S]=0.05 \mathrm{M}$.

validation of the proposed CdS absorption modelization using Mie scattering/absorption theory.

The shape of the size distribution changes at $\omega_{\mathrm{o}}=7.5$ and $\omega_{\mathrm{o}}=15$. Also the trends of $\mathrm{f}_{\mathrm{k}}$ change for these $\omega_{0}$ values. We conclude that the nanoparticles deviate from spherical shape leading to different oscillator strengths (factors $\mathrm{f}_{\mathrm{k}}$ ).

In Figure 2 the photoluminescence of dried agarose gels with embedded CdS particles is plotted for two different SDS concentrations. Two emission bands are observed that can be ascribed to charge recombination $(\sim 520 \mathrm{~nm})$ and defects/trap emission $(\sim 700 \mathrm{~nm}) .{ }^{9}$ It is seen that the increase of $\mathrm{CdS}$ content in the gel clearly increases the relative amount of charge recombination emission.

In FIgUREs 3 and 4 the corresponding excitation spectra, taken at $\sim 520 \mathrm{nnm}$ and $\sim 650 \mathrm{~nm}$ emission, are plotted together with fitted spectra using Mie scattering/absorption theory together with a log-normal ${ }^{10}$ size distribution. In the insets, the corresponding size distributions are shown. In TABLE 2 the corresponding mean particle size and the oscillator strengths factors $\left(\mathrm{f}_{\mathrm{k}}\right)$ are tabulated.

The size distribution of the nanoparticles is broader when $\lambda_{\mathrm{em}}=650 \mathrm{~nm}$ than when $\lambda_{\mathrm{em}}=520 \mathrm{~nm}$, but 
TABLE 2. Fitting of CdS absorption spectra obtained by agarose gel electrophoresis templating (method 2)

\begin{tabular}{lccccccccc}
\hline [SDS $]$ & $\lambda_{\mathrm{em}}(\mathrm{nm})$ & $\mathrm{R}_{\mathrm{m}}{ }^{a}(\mathrm{~nm})$ & $\mathrm{R}_{\max }(\mathrm{nm})$ & $\mathrm{H}(\mathrm{nm})$ & $\mathrm{f}_{2} / \mathrm{f}_{1}$ & $\mathrm{f}_{3} / \mathrm{f}_{1}$ & $\mathrm{f}_{4} / \mathrm{f}_{1}$ & $\mathrm{f}_{5} / \mathrm{f}_{1}$ & $\mathrm{f}_{6} / \mathrm{f}_{1}$ \\
\hline $0.05 \%$ & 520 & 1.69 & 1.66 & 0.34 & 3.13 & $\sim 0$ & 4.68 & 3.82 & 1.35 \\
$0.05 \%$ & 650 & 1.77 & 1.52 & 1.3 & 0.51 & $\sim 0$ & 4.85 & $\sim 0$ & $\sim 0$ \\
$0.5 \%$ & 520 & 1.55 & 1.51 & 0.36 & 1.88 & $\sim 0$ & 10.1 & 1.43 & 0.00012 \\
$0.5 \%$ & 650 & 1.65 & 1.61 & 0.43 & 1.72 & $\sim 0$ & 0.000011 & $\sim 0$ & $\sim 0$ \\
\hline
\end{tabular}

${ }^{a}$ Mean particle size in the $1-5 \mathrm{~nm}$ range, calculated from a Log-normal distribution with $\mathrm{R}_{\max }$ the peak size, $\mathrm{H}$ the peak half-width and a fixed asymmetry factor of 1.2.

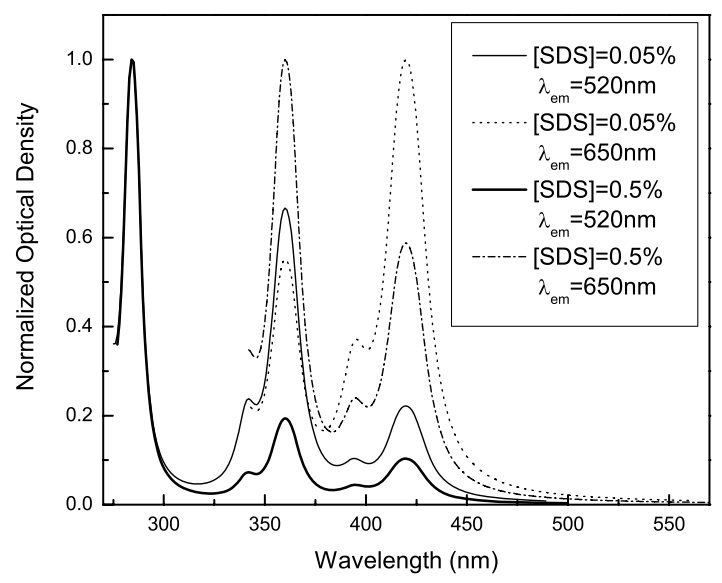

FIGURE 5. Calculated absorption spectra of monodisperse $2 \mathrm{~nm}$ CdS nanoparticles.

that is not the case when the SDS concentration is high. This indicates that SDS promotes the uniformity of the nanoparticles. It was also observed (data not shown) that when SDS concentration is high the nanoparticles obtained with excess $\mathrm{S}^{2-}$ have many more defects. From the obtained size distributions (FIGS. 3 and 4), particles emitting through defect states seem to be distinct from those emitting through charge recombination and correspond to larger particle sizes. We can also conclude that high SDS concentration results in nanoparticles with lower sizes. In gel templating the particles are expected to deviate from sphericity. The observed variations in oscillator strengths (factors $\mathrm{f}_{\mathrm{k}}$ ) should be related to particle shape variations, although there is some covariation between the size distribution and the $\mathrm{f}_{\mathrm{i}}$ factors. On the other hand, this covariation can also influence the recovered particle size distribution. FIGURE 5 plots the calculated absorption spectra of $2 \mathrm{~nm}$ size monodisperse nanoparticles using the $f_{k}$ values obtained from the fitting of the excitation spectra of CdS particles in agarose gels. Comparing the absorption in the $420 \mathrm{~nm}$ region, an increase is observed when $\lambda_{\text {em }}$ goes from $520 \mathrm{~nm}$ to $650 \mathrm{~nm}$. Thus the observed red-shift of the excita- tion spectra when $\lambda_{\mathrm{em}}$ goes from $520 \mathrm{~nm}$ to $650 \mathrm{~nm}$, can be explained by an increase in particle size but also by variations in $f_{k}$ values that influence the edge of the excitation spectrum. This covariation can only be avoided either by experimental measurement of the particle sizes or by theoretical calculations of the oscillator strengths at higher quantum confinement electron energies.

\section{Conflict of Interest}

The authors declare no conflicts of interest.

\section{References}

1. Gizeron, J. \& M.P. Pileni. 1995. Solid solution of $\mathrm{Cd}_{y} \mathrm{Zn}_{1-y} \mathrm{~S}$ nanosize particles made in reverse micelles. J. Phys. Chem. 99: 17410-17416.

2. Grzybowski, B.A., K.J.M. Bishop, C.J. Campbell, et al. 2005. Micro- and nanotechnology via reaction-diffusion. Soft Matter 1: 114-128.

3. Fonoberov, V.A., E.P. Pokatilov \& A.A. Balandin. 2002. Exciton states and optical transitions in colloidal CdS quantum dots: shape and dielectric mismatch effects. Phys. Rev. B 66: 085310.

4. Yu, W.W., L. QU, W. Guo \& X. Peng. 2003. Experimental determination of the extinction coefficient of CdTe, CdSe and CdS nanocrystals. Chem. Mater. 15: 2854-2860.

5. a)Grieve, K., P. Mulvaney \& F. Grieser. 2000. Synthesis and electronic properties of semiconductor nanoparticles/quantum dots. Curr. Opin. Colloid. Interface Sci. 5: 168-172. b) Stupp, S.I. \& P.V. Braun. 1997. Molecular manipulation of microstructures: biomaterials, ceramics and semiconductors. Science 277: 1242 1247. c) Rao, G.N.R., A. Govindaraj, F. Leonard Deepak, et al. 2001. Surfactant-assisted synthesis of semiconductor nanotubes and nanowires. Appl. Phys. Lett. 78: 1853 1855.

6. Rossetti, R., J.L. Ellison, J.M. Gibson \& L.E. Brus. 1984. Size effects in the excited electronic states of small colloidal CdS crystallites. J. Chem. Phys. 80: 4464 4469 .

7. Link, S. \& M.A. El-SAYed. 2000. Shape and size dependence of radiative, non-radiative and photothermal 
properties of gold nanocrystals. Int. Rev. Phys. Chem. 19: 409-453.

8. Pileni, M.P. 1993. Reverse micelles as microreactors. J. Phys. Chem. 97: 6961-6973.

9. Liu, B., G.Q. Xu, L.M. Gan \& C.H. Chew. 2001. Photoluminescence and structural characteristics of CdS nanoclusters synthesized by hydrothermal microemulsion. J. Appl. Phys. 89: 10591063.

10. Siano, D.B. \& D.E. Metzler. 1969. Band shapes of the electronic spectra of complex molecules. J. Chem. Phys. 51: 1856-1861. 